\title{
COMPORTAMIENTO DE LA CORROSIÓN DEL ACERO AISI 304 EMBEBIDO EN CONCRETOS ECOLÓGICOS TERNARIOS BASE CENIZA DE CAÑA DE BAGAZO DE AZÚCAR Y HUMO DE SÍLICE EXPUESTOS A SULFATOS
}

\author{
H. A. ARIZA-FIGUEROA ${ }^{1,2}$, R. CROCHE ${ }^{3}$, F. ALMERAYA-CALDERÓN ${ }^{4}$, \\ F. ESTUPIÑNN ${ }^{4}$, C. GAONA-TIBURCIO ${ }^{4}$, A. C. RAMÍREZ-GARCÍA ${ }^{5}$, \\ R. VILLEGAS-APAEZ ${ }^{6}$, M. A. BALTAZAR-ZAMORA ${ }^{7}$, F. J. OLGUÍN COCA ${ }^{8}$ \\ ${ }^{1}$ Universidad Veracruzana, Doctorado en Ingeniería, FIME, Xalapa, Veracruz, México \\ ${ }^{2}$ GEOTEST S.A. de C.V. - Xalapa, Veracruz, México \\ ${ }^{3}$ Universidad Veracruzana, FIME, Xalapa, Veracruz, México \\ ${ }^{4}$ Universidad Autónoma de Nuevo León. FIME - CIIIA., San Nicolás de los Garza, Nuevo León, México \\ ${ }^{5}$ Universidad Veracruzana, Facultad de Ingeniería, Cd. Mendoza, Veracruz, México \\ ${ }^{6}$ Ingenio Mahuixtlán S.A de C.V, Loc., Mahuixtlán, Coatepec, Veracruz, México \\ ${ }^{7}$ Universidad Veracruzana, Facultad de Ingeniería Civil - Xalapa, Circ. G. Aguirre Beltrán S/N, \\ Lomas del Estadio, CP 91000, Xalapa, Veracruz, México. \\ ${ }^{8}$ Universidad Autónoma del Estado de Hidalgo, Pachuca, México
}

\begin{abstract}
RESUMO
La corrosión del acero de refuerzo es la principal causa del deterioro prematuro en estructuras de concreto. Esta investigación estudia el comportamiento de la corrosión del acero AISI 304 embebido en concretos ecológicos ternarios, con sustitución parcial del Cemento Portland (CPC), combinación de Ceniza de Bagazo de Caña de Azúcar (CBCA) y Humo de Sílice (HS), expuestos al 3.5\% de Na2SO4. La evaluación electroquímica se realizó por 6 meses, mediante técnicas de Potenciales de Corrosión -ASTM C-876-15- y Resistencia a la Polarización Lineal -ASTM G59-. Los resultados indican alta resistencia a la corrosión del acero AISI 304 durante el proceso de monitoreo y niveles de corrosión despreciables, se identifica mayor protección en el concreto con sustitución del 20\% de CPC. Palabras clave: Corrosión, Concretos Ecológicos Ternarios, CBCA, HS, Na2SO $\mathrm{SO}_{4}$ AISI 304.
\end{abstract}

\begin{abstract}
Corrosion of reinforcing steel is the main cause of premature deterioration in concrete structures. This research studies the corrosion behavior of AISI 304 steel embedded in ternary ecological concrete, with partial replacement of Portland Cement (CPC), combination of Sugar Cane Bagasse Ash (SCBA) and Silica Fume (SF), exposed to 3.5\% Na2SO4. The electrochemical evaluation was performed for 6 months, using techniques of Corrosion Potentials -ASTM C-876-15- and Resistance to Linear Polarization -ASTM G59-. The results indicate high resistance to corrosion of the AISI 304 steel during the monitoring process and negligible corrosion levels, greater protection is identified in the concrete with $20 \%$ CPC replacement.
\end{abstract}

Keywords: Corrosion, Ternary Ecological Concrete, SCBA, SF, $\mathrm{Na}_{2} \mathrm{SO}_{4}$, AISI 304. 


\section{RESUMO}

A corrosão do aço de reforço é a principal causa da deterioração prematura das estruturas de concreto. Esta pesquisa estuda o comportamento corrosivo do aço AISI 304 embebido em concreto ecológico ternário, com substituição parcial do cimento Portland (CPC), combinação de cinzas de bagaço de cana de açúcar (CBCA) e fumaça de sílica (FS), expostos a 3,5\% de $\mathrm{Na}_{2} \mathrm{SO}_{4}$. A avaliação eletroquímica foi realizada por 6 meses, utilizando técnicas de Potenciais de Corrosão -ASTM C-876-15- e Resistência a Polarização Linear -ASTM G59-. Os resultados indicam alta resistência à corrosão do aço AISI 304 durante o processo de monitoramento e níveis insignificantes de corrosão, maior proteção é identificada no concreto com $20 \%$ de substituição de CPC.

Palabras-chave: Corrosão, Concreto Ecológico Ternário, CBCA, FS, $\mathrm{Na}_{2} \mathrm{SO}_{4}$, AISI 304.

\section{INTRODUCCIÓN}

Es reconocido a nivel mundial que el problema de la corrosión del acero de refuerzo en estructuras de concreto reforzado es tanto económico como social, problema de miles de millones de dólares (Troconis de Rincón et. al, 2016, García D.L., 2019) y en casos causante de pérdidas humanas por colapso de dichas estructuras. La corrosión del acero de refuerzo es la causa más importante del deterioro prematuro de las estructuras de concreto reforzado siendo el ion cloruro considerado el más agresivo o los medios marinos en general, así lo han demostrado diversas investigaciones (Landa A.E., 2018; Landa A., 2019; Landa A.E., 2018; Landa A., 2019). En un estudios realizado en E.U.A se estimó que más de 244,000 puentes de concreto reforazdo se encontraban dañados por corrosión del acero de refuerzo, (Baltazar, 2016; Belleze, 2006; Landa A.E., 2018; Santiago, 2016). Uno de los agentes agresivos que es de los causantes del daño por corrosión en las estructuras de concreto reforzado son los sulfatos, de sodio, de magnesio, etc. (Santiago, 2016; Landa A.E., 2018; Ariza A.H., 2019; Garcia D.L., 2019, Ruiz M., 2019).; las estructuras más expuestas a este tipo de ataque son las hidráulicas, como canales y tuberías, así como los cimientos en torres de transmisión y pavimentos de carreteras De acuerdo a lo anterior y debido a que se ha demostrado las características puzolánicas potenciales de la CBCA (Chusilp et. al, 2009, Cordeiro et. al, 2008; Cordeiro et. al, 2009; Ganesan et. al., 2007), se presenta la presente investigación, que evalúa el comportamiento de la corrosión en concretos ecológicos ternarios a base de combinaciones de 10, 20 y 30\% de Ceniza de Bagazo de Caña de Azúcar (CBCA) y humo de sílice (HS) como sustitución del Cemento Portland Compuesto (CPC ), para la evaluación de la corrosión se embebieron en los especímenes de estudio barras de acero inoxidable AISI 304 y AISI 1018. Se presentan los resultados de $\mathrm{E}_{\text {corr }}$ e $\mathrm{I}_{\text {corr }}$ de más de 180 días de exposición a un medio sulfatado (solución al $3.5 \%$ de $\mathrm{Na}_{2} \mathrm{SO}_{4}$ ) como medio agresivo y en agua potable como medio de referencia o de control, el propósito es contribuir con información para poder construir estructuras resistentes a la corrosión por sulfatos además de desarrollar concretos sustentables o ecológicos que representen un beneficio para el desarrollo de nuestras sociedades, la utilizar un residuo que es considerado en el estado de Veracruz como basura.

\section{PROCEDIMIENTO}

En la presente investigación se realiza el análisis del comportamiento de la corrosión del acero inoxidable AISI 304 en comparación con el acero al carbono AISI 1018, como refuerzo en especímenes de concretos elaborados con sustitución parcial de CPC 30R por Ceniza de Bagazo de Caña de Azúcar (CBCA) y Humo de Sílice (HS), en 10, 20 y 30\%, los especímenes fueron expuestos a una solución al $3.5 \%$ de $\mathrm{Na}_{2} \mathrm{SO}_{4}$, como medio agresivo. Las técnicas electroquímicas empleadas en el presente estudio fueron las de Potencial de Media Celda, (ASTM C-876-15) y Resistencia a la Polarización LPR (ASTM G59). 


\subsection{Dosificación y Proporcionamiento de las Mezclas de Concreto}

La dosificación de las mezclas se utilizó el método del Instituto Americano del Concreto ACI 211.1 [ACI, 2004], metodología que considera el revenimiento, la resistencia a la compresión de diseño, y las características físicas de los agregados, las cuales se realizaron de acuerdo a las normas ASTM [ASTM, 2007; ASTM, 2014; ASTM, 2015; ASTM, 2015; ASTM, 2016]. La Tabla 1, resume las características físicas de los agregados gruesos y finos que se utilizaron en la presente investigación.

Tabla 1. Ccaracterización física de los agregados.

\begin{tabular}{|c|c|c|}
\hline Propiedades físicas de los materiales & $\begin{array}{c}\text { Agregado } \\
\text { grueso }\end{array}$ & Agregado fino \\
\hline $\begin{array}{c}\text { Densidad relativa aparente saturada y } \\
\text { superficialmente seca (Dr SSS) gr/cm }\end{array}$ & 2.60 & 2.20 \\
\hline Masa Volumétrica Suelta (MVS) Kg/m 3 & 1332.00 & 1442.00 \\
\hline Absorción \% (A) & 1.70 & 1.80 \\
\hline Módulo de finura & $19 \mathrm{~mm}$ & 2.94 \\
\hline Tamaño nominal & & \\
\hline
\end{tabular}

Se utilizó la relación agua/cemento $=0.65$. La mezcla de referencia $(\mathrm{REF})$ es a base de $100 \%$ de cemento portland compuesto (CPC). En las mezclas continuas se realizaron en sustituciones parciales de cemento portland de $10 \%$ a $30 \%$, estos porcentajes se formaron por $50 \%$ de cada adición utilizando ceniza de bagazo de caña de azúcar (SCBA) y humo de sílice (FS). La tabla 2 presenta la dosificación para las cuatro mezclas de estudio, la mezcla control y las tres mezclas de concretos ecológicos ternarios.

Donde:

REF.- Mezcla 100\% CPC, 05,10,15 SCBA y/o FS.- Porcentaje de sustitución de Ceniza de Bagazo de Caña de Azúcar y Humo de Sílice.

Tabla 2. Dosificación de los especímenes de estudio para $1 \mathrm{~m}^{3}$.

\begin{tabular}{|c|c|c|c|c|c|c|}
\hline Mezcla & $\begin{array}{c}\text { Cemento } \\
\text { (kg) }\end{array}$ & $\begin{array}{c}\text { Ceniza de } \\
\text { Bagazo de } \\
\text { Caña }(\mathbf{K g})\end{array}$ & $\begin{array}{c}\text { Humo } \\
\text { de Sílice } \\
(\mathbf{k g})\end{array}$ & $\begin{array}{c}\text { Agua } \\
(\mathbf{K g})\end{array}$ & $\begin{array}{c}\text { Agregad } \\
\text { o fino } \\
(\mathbf{K g})\end{array}$ & $\begin{array}{c}\text { Agregado } \\
\text { grueso } \\
(\mathbf{k g})\end{array}$ \\
\hline REF & 314.61 & - & - & 197.79 & 770.11 & 886.00 \\
\hline 10(05SCBA05FS) & 284.06 & 15.77 & 15.77 & 200.60 & 770.76 & 888.64 \\
\hline 20(10SCBA10FS) & 252.51 & 31.55 & 31.55 & 302.16 & 772.64 & 884.22 \\
\hline 30(15SCBA15FS) & 220.96 & 43.35 & 43.35 & 206.71 & 772.55 & 880.73 \\
\hline
\end{tabular}

\subsection{Caracterización del Concreto Fresco y Endurecido}

De acuerdo a los ensayos de la normativa ONNCCE y ASTM, se determinaron las características del concreto en estado fresco Revenimiento [ONNCCE, 2010], Temperatura [ASTM, 2008], Masa volumétrica [ONNCCE, 2010] y su Resistencia a compresión [ONNCCE, 2014], la tabla 3 presenta dichos resultados. 
Tabla 3. Propiedades físicas y mecánicas de las mezclas de concreto.

\begin{tabular}{|c|c|c|c|c|}
\hline Mezcla & $\begin{array}{c}\text { Temperatura } \\
\left({ }^{\circ} \mathbf{C}\right)\end{array}$ & $\begin{array}{c}\text { Revenimiento } \\
(\mathbf{c m})\end{array}$ & $\begin{array}{c}\text { Masa } \\
\text { Unitaria } \\
\left(\mathbf{k g} / \mathbf{m}^{\mathbf{3}}\right)\end{array}$ & $\begin{array}{c}\mathbf{F}^{\prime} \mathbf{c} \\
\left(\mathbf{k g} / \mathbf{c m}^{\mathbf{2}}\right)\end{array}$ \\
\hline REF & 24.00 & 7.00 & 2345.83 & 299.37 \\
\hline 10(05SCBA05FS) & 23.50 & 6.00 & 2307.29 & 335.39 \\
\hline 20(10SCBA10FS) & 23.50 & 5.50 & 2301.04 & 288.89 \\
\hline 30(15SCBA15FS) & 22.50 & 5.00 & 2276.04 & 232.79 \\
\hline
\end{tabular}

\subsection{Características de las barras de refuerzo}

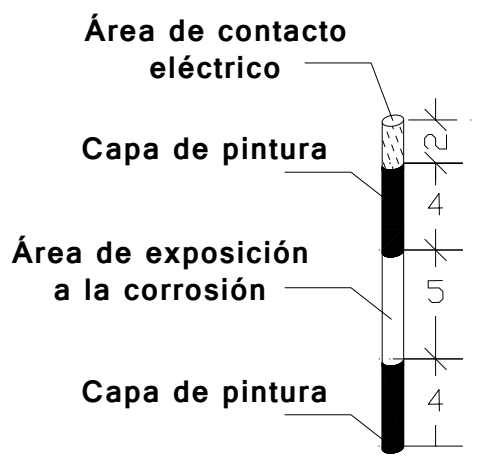

Figura 1. Características barras acero inoxidable 304, 316 y AISI 1018 (cotas en cm).

En cada espécimen se embebieron tres barras de 3/8" de diámetro, una de acero inoxidable 304, la segunda de acero inoxidable 316 y la tercera de acero AISI 1018, a estas tres barras se utilizaron como electrodos de trabajo (ET) para la evaluación del comportamiento de la corrosión (monitoreo de $\mathrm{E}_{\text {corr }} \mathrm{e}$ $I_{\text {corr }}$, (Santiago, 2013). Como electrodo auxiliar se utilizó una barra de acero inoxidable 304 de 1/16", ver figura 1.

\subsection{Nomenclatura de especímenes}

Para la facilitar la identificación de los especímenes se asignó nomenclaturas, para los ensayes de resistencia a la compresión, resistividad y módulo de elasticidad expuestos a los medios agresivos, sulfato de sodio y sulfato de magnesio.

Tabla 4. Nomenclatura de especímenes de ensaye a compresión y Resistividad.

\begin{tabular}{|c|c|}
\hline Nomenclatura & Ensayo \\
\hline MC-304 y MC-1018 & $\mathrm{I}_{\text {corr }} 180$ días \\
\hline M1-304 у M1-1018 & $\mathrm{I}_{\text {corr }} 180$ días \\
\hline M2-304 y M2-1018 & $\mathrm{I}_{\text {corr }} 180$ días \\
\hline M3-304 y M3-1018 & $\mathrm{I}_{\text {corr }} 180$ días \\
\hline
\end{tabular}

Para la facilitar la identificación de los especímenes se realizaron nomenclaturas, para los ensayes de resistencia a la compresión, resistividad y módulo de elasticidad expuestos a los medios agresivos, sulfato de sodio y sulfato de magnesio.

Donde:

REF.- Mezcla 100\% CPC. 
05,10,15 SCBA y/o FS.- porcentaje de sustitución de Ceniza de Bagazo de Caña de Azúcar y Humo de Sílice.

MC.- Medio control, agua potable

M1, 2, 3.- Medio de exposición, sulfato de sodio

Tabla 5. Nomenclatura de especímenes del ensaye de Módulo de elasticidad.

\begin{tabular}{|c|c|}
\hline Nomenclatura & Ensayo \\
\hline MC-304 у MC-1018 & $\mathrm{E}_{\text {corr }} 180$ días \\
\hline M1-304 у M1-1018 & $\mathrm{E}_{\text {corr }} 180$ días \\
\hline M2-304 у M2-1018 & $\mathrm{E}_{\text {corr }} 180$ días \\
\hline M3-304 у M3-1018 & $E_{\text {corr }} 180$ días \\
\hline
\end{tabular}

Donde:

REF.- Mezcla $100 \%$ CPC.

05,10,15 SCBA y/o FS.- porcentaje de sustitución de Ceniza de Bagazo de Caña de Azúcar y Humo de Sílice.

MC.- Medio control, agua potable

M1, 2, 3.- Medio de exposición, sulfato de sodio

\subsection{Celda electroquímica}

Los especímenes de estudio fueron cubos de $15 \mathrm{~cm}$ por lado, la celda electroquímica fue de acuerdo a la norma ASTM G59 (ASTM, 2009), para determinar la técnica electroquímica de resistencia a la Polarización Lineal (RPL), con un arreglo de tres electrodos, un electrodo de referencia (ER:Cobre Sulfato de Cobre), Electrodo de Auxiliar y el Electrodo de Trabajo (ET), el arreglo de la celda se puede observar en la figura 2.

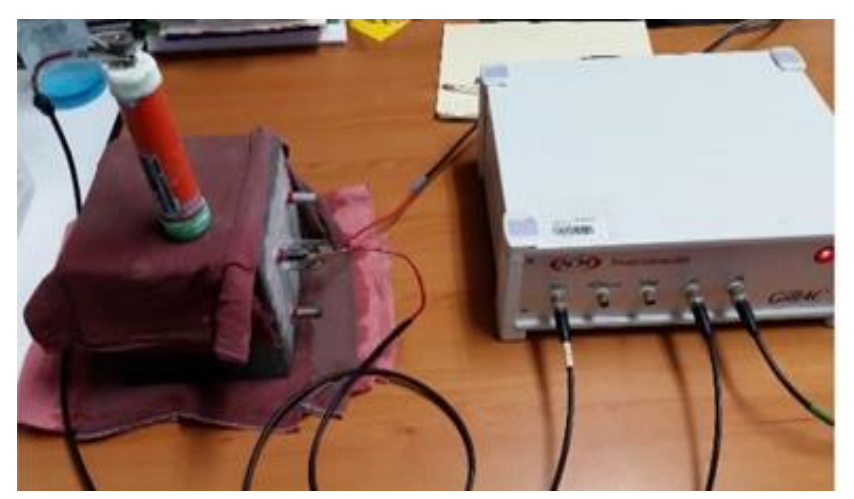

Figura 2. Celda electroquímica para el monitoreo de la corrosión

\section{RESULTADOS}

Los resultados de los ensayes electroquímicos de los especímenes a base de concretos ternarios se muestran a continuación.

\subsection{Potencial de Corrosión}

Para interpretar los resultados obtenidos del Potencial de Corrosión de cada uno de los especímenes de prueba, se analizaron acorde a norma ASTM C876-15 [ASTM, 2015], agregando un rango más de acuerdo a la literatura [Won-Song, 2007], lo anterior se resume en la tabla 6. 
Tabla 6. Potenciales de corrosión en concreto reforzado.

\begin{tabular}{|c|c|}
\hline \multicolumn{2}{|c|}{ Potenciales de corrosión mV vs Cu/CuSO4 } \\
\hline$<-500$ & Corrosión severa \\
\hline$<-350$ & $\begin{array}{c}90 \% \text { de Probabilidad de } \\
\text { Corrosión }\end{array}$ \\
\hline$-350 \mathrm{a}-200$ & Incertidumbre \\
\hline$>-200$ & $\begin{array}{c}10 \% \text { de Probabilidad de } \\
\text { Corrosión }\end{array}$ \\
\hline
\end{tabular}

\section{2 $E_{\text {corr }}$ de barras de acero inoxidable 304 y acero 1018}

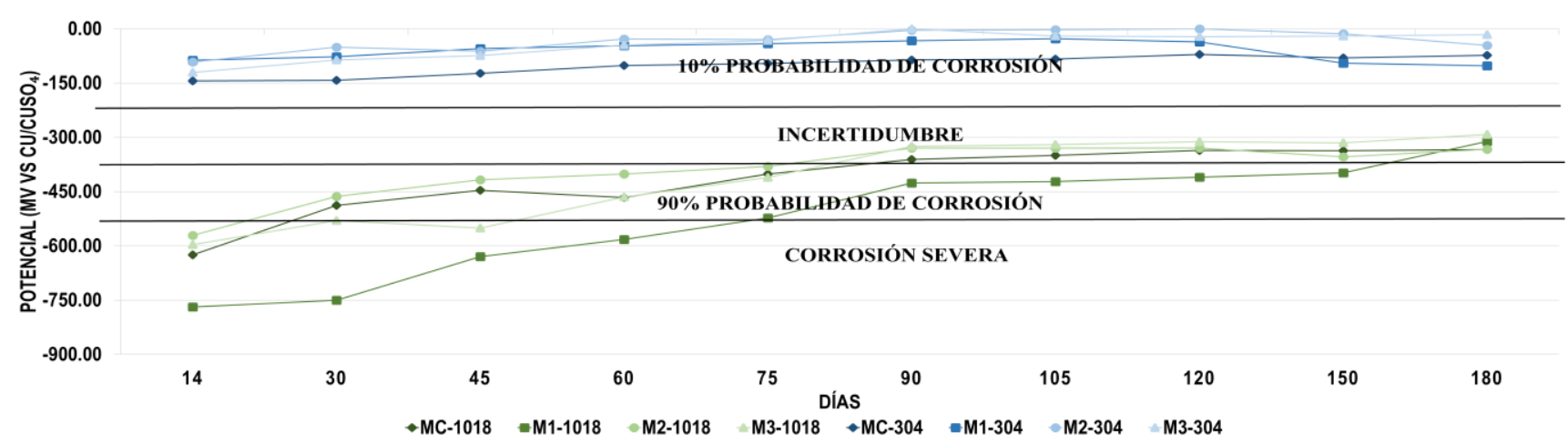

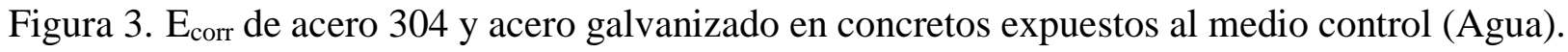

El acero inoxidable 304 embebido en los 4 especímenes de estudio, presentan en la etapa de curado potenciales de $-144 \mathrm{mV}$ a $-86 \mathrm{mV}$ a los 90 días al estar expuestos al medio control, agua potable, lo que nos indica un potencial de corrosión del 10\%, se mantiene una tendencia de valores hasta los 180 días. En el análisis de los valores del acero galvanizado, durante los días de curado se presentan valores que tienden a una corrosión severa del $-600 \mathrm{mV}$ a $-463 \mathrm{mV}$, sin embargo, a los 90 días de exposición los valores comienzan a ser más positivos oscilando en los $-325 \mathrm{mV}$, manteniendo este comportamiento durante los 3 meses siguientes.

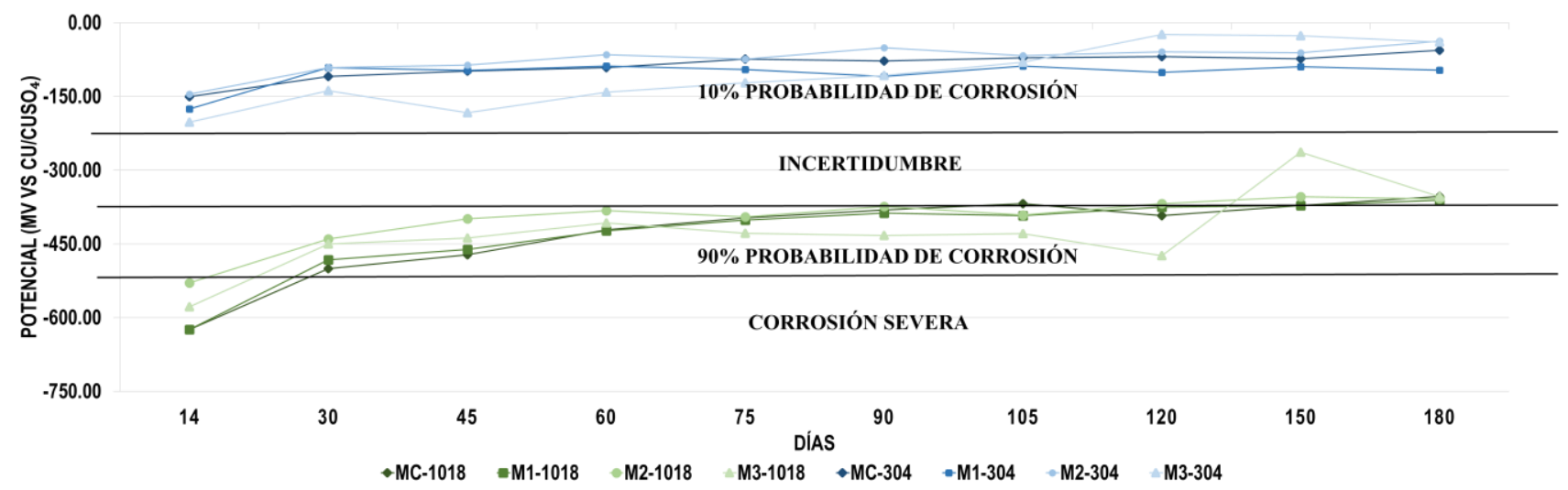

Figura 4. $\mathrm{E}_{\text {corr }}$ de acero 304 y acero galvanizado en concretos expuestos a sulfato de sodio $\left(\mathrm{Na}_{2} \mathrm{SO}_{4}\right)$.

En la Figura 4, presenta el comportamiento del acero inoxidable 304 en presencia de sulfato de sodio al $3.5 \%$, se observa en los especímenes con 100\% de CPC, de 10\% al 30\% de CBCA y HS, presentan la tendencia a valores más negativos en la etapa de curado, para los monitores a los 90 días, los valores empiezan a ser más positivos, con un rango de $-109 \mathrm{mV}$ a $-51 \mathrm{mV}$, presentando una mejor protección COMPORTAMIENTO DE LA CORROSIÓN DEL ACERO 
a la corrosión, lo que de acuerdo a la norma ASTM C-876 indica un 10\% de probabilidad de corrosión. Los especímenes que recubren el acero galvanizado después de la etapa de curado, los resultados empiezan a oscilar entre los $-500 \mathrm{mV}$ a los $-353 \mathrm{mV}$, manteniendo el potencial de corrosión en los valores del 90\%. Si bien concreto con sustitución parcial de CPC al 30\% visualiza un momento de incertidumbre a los 150 días, su comportamiento es inestable, caso contrario de lo que sucede con el concreto en $20 \%$ de sustitución del CPC, que presenta a lo largo de 150 días un comportamiento estable y empezando a arrojar valores más positivos.

\subsection{Cinética de Corrosión ( $\mathbf{I}_{\text {corr }}$ )}

Los resultados obtenidos de la cinética de corrosión $\mathrm{I}_{\text {corr }}$, fueron interpretados de acuerdo al criterio del Manual de la red Durar (O. Trocónis et. al., 1997).

Tabla 7. Nivel de corrosión de acuerdo a la Icorr.

\begin{tabular}{|c|c|}
\hline $\begin{array}{c}\text { Velocidad de Corrosión } \\
\left(\mathbf{i}_{\text {icorr }} \boldsymbol{\mu} \mathbf{A} / \mathbf{c m}^{2}\right.\end{array}$ & Nivel de Corrosión \\
\hline$<0.1$ & Despreciable \\
\hline $0.1-0.5$ & Moderada \\
\hline $0.5-1$ & Elevada \\
\hline$>1$ & Muy elevada \\
\hline
\end{tabular}

En la siguiente tabla, se visualiza, la velocidad de corrosión que presentaron los especímenes de estudios en los primeros 180 días de exposición al Medio Control, como puede observarse, los especímenes con acero inoxidable presentaron un nivel de corrosión despreciable, se relaciona dicho comportamiento a que están expuestos a un medio no agresivo, sin embargo, el nivel de corrosión en aceros 1018 se presenta como de moderada a elevada. Se espera que al presentarse en medio más agresivos este comportamiento se vea modificado.

Tabla 8. Intensidad de Corriente especímenes Medio Control (Evaluación a los 180 días)

\begin{tabular}{|c|c|c|}
\hline Espécimen & $\begin{array}{c}(\mathbf{I} \text { corr }) \\
\boldsymbol{\mu A} / \mathbf{c m}^{2}\end{array}$ & $\begin{array}{c}\text { Nivel de } \\
\text { Corrosión }\end{array}$ \\
\hline MC-1018 & 0.172 & Moderada \\
\hline M1-1018 & 0.335 & Moderada \\
\hline M2-1018 & 0.847 & Elevada \\
\hline M3-1018 & 0.027 & Despreciable \\
\hline MC-304 & 0.027 & Despreciable \\
\hline M1-304 & 0.029 & Despreciable \\
\hline M2-304 & 0.038 & Despreciable \\
\hline M3-304 & 0.013 & Despreciable \\
\hline
\end{tabular}

La velocidad de corrosión que presentaron los especímenes de estudios en los primeros 180 días de exposición a una solución al 3.5\% de Na2SO4 como medio agresivo, se muestran en la siguiente tabla, se tiene que los especímenes con aceros inoxidables 304, presentan un excelente comportamiento contra la corrosión con valores entre 0.006 a $0.013 \mu \mathrm{A} / \mathrm{cm} 2$, valores despreciables. Para los aceros 1018 los valores empiezan a mostrar un mejor comportamiento ante la corrosión, tal como lo marca la literatura evidenciando el beneficio del uso de este residuo agroindustrial, resultados que concuerdan con la literatura (A.E. Landa-Gómez, 2018). 
Tabla 9. Intensidad de Corriente especímenes, medio de exposición, sulfato de sodio $\mathrm{Na}_{2} \mathrm{SO}_{4}$ (Evaluación a los 180 días)

\begin{tabular}{|c|c|c|}
\hline Espécimen & $\begin{array}{c}\left(\mathbf{I}_{\text {corr }}\right) \\
\boldsymbol{\mu A} \mathbf{A} \mathbf{c m}^{2}\end{array}$ & $\begin{array}{c}\text { Nivel de } \\
\text { Corrosión }\end{array}$ \\
\hline MC-1018 & 0.021 & Despreciable \\
\hline M1-1018 & 0.028 & Despreciable \\
\hline M2-1018 & 0.209 & Moderada \\
\hline M3-1018 & 0.207 & Moderada \\
\hline MC-304 & 0.012 & Despreciable \\
\hline M1-304 & 0.013 & Despreciable \\
\hline M2-304 & 0.006 & Despreciable \\
\hline M3-304 & 0.010 & Despreciable \\
\hline
\end{tabular}

\section{CONCLUSIONES}

De acuerdo a los resultados, se muestra que el comportamiento de la corrosión de los aceros inoxidables embebidos en concretos sustentables a base de Ceniza de Bagazo de Caña de Azúcar después de 180 días de exposición a un medio sulfatado (solución al 3.5\% de NaSO4) es excelente, presentando valores de corrosión de despreciables a moderados. Los concretos con sustituciones parciales de cemento portland utilizando acero inoxidable presentan un nivel de corrosión despreciable, en comparación de los concretos con acero galvanizado, lo que indica un beneficio del concreto sustentable a base de Ceniza de Bagazo de Caña de Azúcar.

Con el presente estudio se demostró la viabilidad del uso de la Ceniza de Bagazo de Caña de Azúcar, Humo de sílice y aceros inoxidables, para elaborar concretos sustentables resistentes a sulfatos, los cuales pueden incrementar su eficiencia anticorrosiva, para contribuir a la construcción sustentable de edificaciones de obra civil

\section{AGRADECIMIENTOS}

M.A. Baltazar-Zamora, et. al., agradecen a PRODEP por el Apoyo otorgado por la SEP, al Cuerpo Académico UV-CA-458 "Sustentabilidad y Durabilidad de Materiales para la Infraestructura Civil", en el marco de la Convocatoria 2018 para Fortalecimiento de Cuerpos Académicos con IDCA 28593.

\section{REFERENCIAS}

Ariza H.A., Croche R., Gaona C., Almeraya F., Ramírez A.C., Villegas R., Márquez S., BaltazarZamora M.A.,(2019). "Evaluación de la corrosión del acero galvanizado y acero AISI 304 embebidos en concretos ecológicos con sustitución de CPC por 10, 20 y 30\% de CBCA-HS expuestos a $\mathrm{Na}_{2} \mathrm{SO}_{4}$ al 3.5\%." Memorias del XXXIV Congreso Nacional de la Sociedad Mexicana de Electroquímica, 12th Meeting of the Mexican Section of the Electrochemical Society, SMEQ, Querétaro (México).

ASTM International. (2007), ASTM C29 / C29M-07-Standard Test Method for Bulk Density ("Unit Weight") and Voids in Aggregate.

ASTM International. (2014), ASTM C136 / C136M -14-Standard Test Method for Sieve Analysis of Fine and Coarse Aggregates.

ASTM International. (2015), ASTM C127-15-Standard Test Method for Relative Density (Specific Gravity) and Absorption of Coarse Aggregate.

ASTM International. (2015), ASTM C128-15-Standard Test Method for Relative Density (Specific Gravity) and Absorption of Fine Aggregate. 
ASTM International. (2016), ASTM C33/C33M-16e1-Standard Specification for Concrete Aggregates.

ASTM International. (2015), ASTM C 876-15, Standard Test Method for Corrosion Potentials of Uncoated Reinforcing steel in Concrete.

ASTM International. (2008) ASTM C1064/C1064M - 08 Standard test method for temperature of freshly Mixed Hydraulic-Cement Concrete.

ASTM International. (2009) ASTM G 59-97(2009), Standard Test Method for Conducting Potentiodynamic Polarization Resistance Measurements.

Baltazar-Zamora M.A., Santiago, G., Moreno V., Croche R., De la Garza M., Estupiñan, F., Zambrano, P., Gaona, C. (2016). Electrochemical Behaviour of Galvanized Steel Embedded in Concrete Exposed to Sand Contaminated with NaCl. Int. J. Electrochem. Sci. 11, 10306-10319, doi: 10.20964/2016.12.28. Bellezze T., Malavolta M., Quaranta A., Ruffini N., Roventi G. (2006). Corrosion behaviour in concrete of three differently galvanized steel bars. Cement and Concrete Composites., 28, 246-255.

Chusilp N., Jaturapitakkul C., Kiattikomol K. (2009). Utilization of bagasse ash as a pozzolanic material in concrete. Construction and Building Materials, 23, 3352-3358.

Comité ACI 211.1, ACI. (2004) "Proporcionamiento de Mezclas, Concreto Normal, Pesado y Masivo ACI 211.1”, IMCYC, México, D.F.

Cordeiro G.C., Toledo R.D., Tavares E.R. (2008). Pozzolanic activity and filler effect of sugar cane bagasse ash in Portland cement and lime mortars. Cement and Concrete Composites, 30, 410-418.

Cordeiro, G. C., Toledo, R. D., \& Fairbairn, E. M. (2009). Effect of calcination temperature on the pozzolanic activity of sugar cane bagasse ash. Construction and Building Materials, 23, 3301-3303.

Ganesan, K., Rajagopal, K., \& Thangavel, K. (2007). Evaluation of bagasse ash as supplementary cementitious material. Cement and Concrete Composites, 29, 515-524.

García D.L, Cabral J.A, Gaona C., Almeraya F., Ramírez A.C., Croche R., Baltazar-Zamora M.A.,(2019) "Influencia del medio ambiente de exposición y tipo de curado en la cinética de corrosión de concreto reforzado" Memorias del XXXIV Congreso Nacional de la Sociedad Mexicana de Electroquímica, 12th Meeting of the Mexican Section of the Electrochemical Society, SMEQ, Querétaro (México).

García D.L, Cabral J.A, Gaona C., Almeraya F., Ramírez A.C., Villegas R., Marquez S., BaltazarZamora M.A.,(2019). "Eficiencia anticorrosiva del acero AISI 304 como refuerzo en concretos ecológicos ternarios base CBCA-HS expuestos a sulfato de magnesio". Memorias del XXXIV Congreso Nacional de la Sociedad Mexicana de Electroquímica, 12th Meeting of the Mexican Section of the Electrochemical Society, SMEQ, Querétaro (México).

Landa A.E., Croche R., Márquez S., Villegas R., Ariza H.A., Estupiñan F., Gaona C., Almeraya F., Baltazar-Zamora M.A. (2018). Corrosion Behavior 304 and 316 Stainless Steel as Reinforcement in Sustainable Concrete Based on Sugar Cane Bagasse Ash Exposed to $\mathrm{Na}_{2} \mathrm{SO}_{4}$, ECS transaction, 84, 179188

Landa A.E., Croche R., Márquez S., Galván R., Gaona C., Almeraya F., Baltazar-Zamora M.A. (2018). Correlation of Compression Resistance and Rupture Module of a Concrete of Ratio $w / c=0.50$ with the Corrosion Potential, Electrical Resistivity and Ultrasonic Pulse Speed, ECS transactions, 84, 217-227 Landa A.E., Croche R., López O.M., Galván R., Cabral J.A., Gaona C., Almeraya F., Baltazar-Zamora M.A. (2018). Corrosion Behavior of AISI 316 Stainless Steel As Reinforcement in Ternary Sustainable Concrete Based on SCBA-SF Exposed in Seawater, ECS Meeting Abstracts, MA2018-02 584-584

Landa A.E., Castillo S., Reyes J.A., Villegas R., Galván R., Cabral J.A., Gaona C., Almeraya F., Baltazar-Zamora M.A. (2018). Evaluation of the Corrosion of AISI 304 Stainless Steel Embedded in Sustainable Concrete with High Volumes of SCBA-SF Exposed in Marine Environment, ECS Meeting abstracts, MA2018-02 587

Landa A., Cabral J., Gaona C., Almeraya F., Ramírez A.C., Ruiz M., Malpica R., Márquez S., BaltazarZamora M.A. (2019) "Evaluación electroquímica del acero inoxidable AISI 316 embebido en concretos 
sustentables base ceniza de bagazo de caña de azúcar expuestos a un medio marino" Memorias del XXXIV Congreso Nacional de la Sociedad Mexicana de Electroquímica, 12th Meeting of the Mexican Section of the Electrochemical Society, SMEQ, Querétaro (México).

Landa A., Landa A., Rangel J.M., Almeraya F., Croche B., Baltazar-Zamora M.A., (2019). "Comportamiento de la corrosión del acero AISI 316 como refuerzo en concretos ternarios sustentables con sustitución parcial de CPC en 10\%, 20\% y 30\% de CBCA-HS inmersos en agua de mar'. Memorias del XXXIV Congreso Nacional de la Sociedad Mexicana de Electroquímica, 12th Meeting of the Mexican Section of the Electrochemical Society, SMEQ, Querétaro (México).

Organismo Nacional de Normalización y Certificación de la Construcción y Edificación, S. C. (2014) "NMX-C-083-ONNCCE-2002: Industria de la Construcción - Concreto - Determinación de la Resistencia a la Compresión de Especímenes - Método de Ensayo", ONNCCE, S.C., México

Organismo Nacional de Normalización y Certificación de la Construcción y Edificación, S. C. (2010) "NMX-C-156-ONNCCE-2010: Industria de la Construcción - Concreto Hidráulico - Determinación Del Revenimiento En El Concreto Fresco", ONNCCE, S.C., México

Organismo Nacional de Normalización y Certificación de la Construcción y Edificación, S. C. (2010) "NMX-C-162-ONNCCE-2010: Industria de la construcción - Concreto hidráulico - Determinación de la masa unitaria, cálculo del rendimiento y contenido de aire del concreto fresco por el método gravimétrico", ONNCCE, S.C., México

Ruíz M., Landa A., Cabral J.A., Gaona C., Almeraya F., Malpica R., Márquez S., Baltazar M.A., (2019) "Influencia del tipo de acero en el comportamiento de la corrosión de concretos sustentables ternarios elaborados con ceniza de bagazo de caña de azúcar y humo de sílice expuestos a sulfato de sodio" Memorias del XXXIV Congreso Nacional de la Sociedad Mexicana de Electroquímica, 12th Meeting of the Mexican Section of the Electrochemical Society, SMEQ, Querétaro (México).

Santiago-Hurtado G., Baltazar-Zamora M.A., Galindo A., Cabral J.A., Estupiñán F., Zambrano Robledo P., Gaona-Tiburcio C. (2013). Anticorrosive Efficiency of Primer Applied in Carbon Steel AISI 1018 as Reinforcement in a Soil Type MH. Int. J. Electrochem. Sci., 8, 8490.

Santiago, G., Baltazar-Zamora M.A., Galván, R., López, L.D., Zapata, F., Zambrano, P., A. Gaona, C. and Almeraya, F. (2016), Electrochemical evaluation of reinforcement concrete exposed to soil type sp contaminated with sulphates. Int. J. Electrochem. Sci. 11,4850-4864, doi: 10.20964/2016.06.31

Santiago-Hurtado, G., Baltazar-Zamora, M.A., Galván-Martínez, R., López, L. D., Zapata G, F., Zambrano, P., Gaona-Tiburcio, C., Almeraya-Calderón, F. (2016), Electrochemical Evaluation of Reinforcement Concrete Exposed to Soil Type SP Contaminated with Sulphates, Int. J. Electrochem. Sci., 11, 4850.

Troconis de Rincón et. al. (2016), "Reinforced Concrete Durability in Marine Environments DURACON Project: Long-Term Exposure". Corrosion, 6 (72), 824.

Trocónis O. et. al. (1997). Manual de Inspección, Evaluación y Diagnóstico de Corrosión en Estructuras de Hormigón Armado. Red DURAR. CYTED. Venezuela. pp.134

Won-Song H. and V. Saraswathy V. (2007), "Corrosion Monitoring of Reinforced Concrete Structures - A Review”. Int. J. Electrochem. Sci., 2, 1-28. 